\title{
La gestión educativa en el desarrollo de la calidad universitaria
}

\section{Educational management in the development of university quality}

Judith Soledad Yangali Vicente

Universidad Nacional de Cañete, Perú

Autor para correspondencia: judithsyv@ gmail.com

Fecha de recepción: 29 de junio de 2016 - Fecha de aceptación: 30 de julio de 2016

\section{Resumen}

La presente investigación estudio en qué medida la gestión educativa mejoro la calidad en la Universidad Nacional de Cañete, encontrándose que, en una adecuada gestión educativa, mejora significativamente al contar con gerentes universitarios competentes para una adecuada gestión de la carrera y garantizar el soporte institucional en la Universidad Nacional de Cañete. Es una investigación básica de tipo aplicada, de carácter exploratorio, según su naturaleza es cuantitativa, según su alcance es longitudinal. El diseño es experimental, de nivel cuasi experimental. La población es de 817 personas y se aplicó en una muestra de 142 personas. Llegando a las siguientes conclusiones: La gestión educativa, considera que los directivos y funcionarios universitarios están tomando conciencia de la importancia del conocimiento y dominio de la gestión estratégica para alcanzar los objetivos institucionales en la universidad y garantizar la calidad educativa.

Palabras claves: gestión, estratégica, resultados, calidad educativa.

\begin{abstract}
This research study to what extent educational management improved the quality of education at the National University of Cañete, finding that proper management education improves significantly by having competent university managers for proper career management and ensuring institutional support the National University of Cañete. It is a basic type applied research, exploratory in nature, according to its nature is quantitative, as its scope is transverse longitudinal. The design is experimental, quasi-experimental level. The population is 817 people and applied in a sample of 71 people. Reached the following conclusions: educational management believes that managers and university officials are becoming aware of the importance of knowledge and mastery of strategic management to achieve corporate goals in college and ensure educational quality.
\end{abstract}

Key words: management, strategic, results, quality of education. 


\section{Introducción}

La presente investigación estudio en qué medida la gestión educativa mejoro la calidad en la Universidad Nacional de Cañete, encontrándose que una adecuada gestión educativa, mejoro significativamente en contar con gerentes universitarios competentes para una adecuada gestión de la carrera y garantizar el soporte institucional en la Universidad Nacional de Cañete. La optimización de la gestión educativa se enfoca en una gestión por competencias y una buena alternativa para el mundo educativo; su eficacia e impacto depende más bien de las capacidades de liderazgo y gestión de los equipos directivos. Y entendiendo a la calidad educativa, como la meta de toda institución que asume un enfoque global de gestión de la institución, hacia la consecución de metas de calidad para todos y con la colaboración de todos los implicados en la institución educativa, en este caso la universidad nacional de Cañete.

Problema

En qué medida la aplicación del programa de innovación "Rumbo al licenciamiento", tiene efectos significativos en la gestión educativa, para la mejora de la calidad educativa en la carrera de administración de la universidad nacional de Cañete.

\section{Objetivo}

Determinar en qué medida la aplicación del programa de innovación "Rumbo al licenciamiento", tiene efectos significativos en la gestión educativa, para la mejora de la calidad educativa en la carrera de administración en la Universidad Nacional de Cañete.

\section{Hipótesis}

La aplicación del programa de innovación "Rumbo al licenciamiento", tiene efectos significativos en la gestión educativa, para la mejora de la calidad educativa en la carrera de administración de la universidad nacional de Cañete.

\section{Justificación}

La investigación busca dar solución a este problema, porque a partir de la aplicación del programa de innovación "Rumbo al licenciamiento", permitirá diseñar y desarrollar un sistema adecuado de gestión educativa de calidad para la Universidad Nacional de Cañete, a través del cumplimiento de las Condiciones Básicas de Calidad que contiene 55 indicadores que serán necesarias para el Licenciamiento definitivo, por lo que se tuvo que trabajar con los agentes de cambio que es el recurso humano de la universidad. Poniendo énfasis en la gestión educativa que desarrollan las autoridades y funcionarios de la universidad, trazándose objetivos institucionales a lograr en la universidad nacional de Cañete, analizando su organización, identificando sus puntos fuertes que contribuyan a ser apoyo de los puntos débiles, priorizando áreas que se reforzaron en el plan de mejora y de acción de implementación para acceder al licenciamiento, a través de la autoevaluación como parte del Programa de innovación "Rumbo al licenciamiento", en la carrera de administración en la Universidad Nacional de Cañete en el año 2015. La investigación es cuasi experimental y podrá ser generalizada a todas las universidades, convirtiéndose por lo tanto en una teoría demostrable y aplicable. 
La presente investigación resulta ser importante, porque servirá como referente, tanto para las universidades públicas o privadas, la eficiencia de la aplicación de un programa de mejora de la calidad educativa que desarrollen los miembros de la comunidad universitaria, a fin de lograr que se identifiquen con su institución y esté debidamente motivados para que puedan prestar sus servicios de la mejor menara y con el mayor esmero posible, hecho que ayudará en la obtención del Licenciamiento en la universidad que la apliquen, por lo que se prestigiarán en la sociedad. Después de lo tratado, se puede desagregar entonces que; el licenciamiento es la verificación del cumplimiento de las condiciones básicas de calidad en las universidades, que permitirá ofrecer una educación de calidad al estudiante, quién ahora es el centro de toda la reforma universitaria planteada a partir de la Ley Universitaria $\mathrm{N}^{\mathbf{o}} 30220$ en el Perú.

El Programa de innovación aplicado en la presente investigación ha considerado el aporte de García (2000) quien señala que la gestión educativa se debe entender al logro de los objetivos y metas educacionales, atendiendo las necesidades básicas de los alumnos, de los padres, de los docentes y de la comunidad toda, en pos de un modelo de país solidario, ético y participativo.

Asimismo, considerando los lineamientos y marco referencial de la UNESCO (2011) precisa que la gestión educativa se compone de cuatro dimensiones: La institucional, pedagógica o didáctica, la administrativa y la socio - humanística o comunitaria. Así también cabe indicar que la gestión educativa propicia en las autoridades académicas la reflexión sobre su función directiva, el análisis de la gestión en la institución proponiendo instrumentos y procedimientos orientados hacia el logro de una Educación de Calidad y enmarcada en los principios de la UNESCO y las Naciones Unidas (p.12). Y tomando los aportes de Guerrero, se entiende que la Gestión Educativa, es la capacidad de generar una reflexión adecuada entre la estructura, la estrategia, los sistemas, el estilo de liderazgo, las capacidades, la gente y los objetivos superiores de la organización considerada, así como la capacidad de articular los recursos de que se dispone de manera de lograr lo que se desea. CINDA (1999) en la gestión nos indica que debe interesar de un modo preeminente que las cosas se hagan, se realicen, se pongan por obra, se solucionen los problemas, esto es indispensable, es condición necesaria aunque no suficiente, ya que al igual que en todo quehacer humano es adecuado pensar antes de actuar. Así, para realizar una buena gestión es conveniente pensar lo que se desea lograr antes de iniciar las acciones y para ello se dispondrá de modelos que ayuden a simplificar la complejidad de la realidad que se enfrente (p.81).

Entonces podemos decir que en términos simples quien está a cargo ya sea el jefe, el presidente, el director, el gerente, o el rector de una organización como la universidad debe ocuparse de su gobierno, y el proceso de gobierno, es decir, su proceso político, en el lenguaje empresarial común y corriente se conoce como gestión estratégica, esta es la responsabilidad principal de quien está a cargo de su gobierno y consiste en: determinar los fines que se persiguen, estructurar la función de gobierno, es decir establecer la configuración institucional; y mantener y desarrollar la convivencia profesional de los colaboradores.

En relación al concepto de calidad, es polivalente, es decir, puede ser analizado desde distintas perspectivas, enfoques o modelos y ha ido evolucionando con el tiempo, generalmente, asociado al ámbito industrial o empresarial. En este ámbito "podría resumirse en tres niveles: el 
nivel o producto relacionado con el control de la calidad; el nivel de proceso o sistema asociado con el sistema de aseguramiento de la calidad; y el nivel de gestión asociado a la calidad total" (Morales, 2010, p. 26). El ministerio de educación a través de la SUNEDU precisa que el servicio educativo universitario de calidad, busca garantizar que todos los jóvenes del país tengan la oportunidad de acceder a una formación integral y de perfeccionamiento continuo, centrado en el logro de un desempeño profesional competente y, en la incorporación de valores ciudadanos que permiten una reflexión académica del país, a través de la investigación. De todo lo tratado en la investigación se desprende que según refiere DIGESU (2015), la calidad se le define como "la congruencia entre los objetivos, resultados y procesos del sistema educativo conforme a las dimensiones de eficacia, eficiencia, pertinencia y equidad" (p.6).

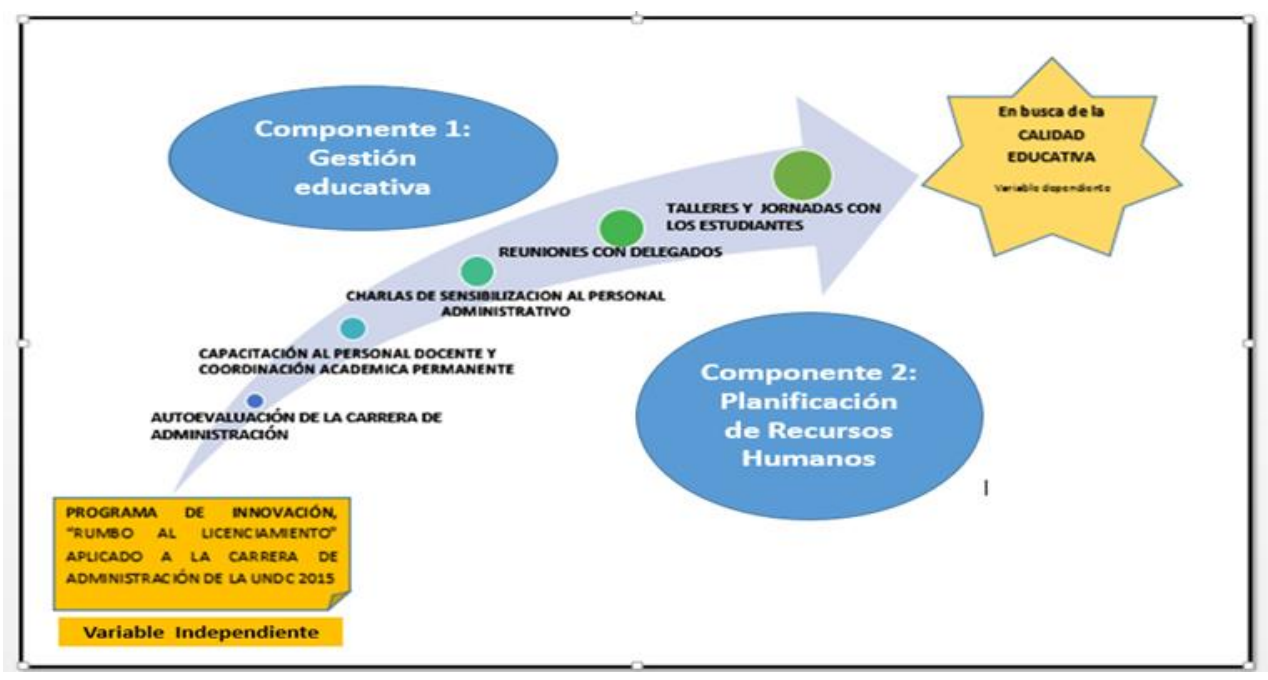

Figura 1. La aplicación del programa de innovación” Rumbo al Licenciamiento" en la Universidad Nacional de Cañete

\section{Método}

En esta investigación se aplicó el método experimental, el tipo de estudio es una investigación de tipo aplicada, es de carácter exploratorio, según su naturaleza es cuantitativa, según su alcance es longitudinal. El diseño es experimental, de nivel cuasi experimental.

\section{La población}

El objeto de estudio está constituido por 817 personas (el universo) de ambos sexos entre docentes, administrativos y estudiantes de la "Universidad Nacional de Cañete" del distrito de San Vicente de la Provincia de Cañete, en el año 2015, tal como se detalla en el siguiente cuadro:

Tabla 1. Distribución de la población docente, administrativa y estudiantes de la "Universidad Nacional de Cañete" del distrito de San Vicente de la Provincia de Cañete, en el año 2015

\begin{tabular}{lll}
\hline Población & Sexo & $\mathrm{N}^{\circ}$ de Personal y Estudiante \\
\cline { 2 - 3 } & $\mathrm{M}$ \\
\hline
\end{tabular}




\begin{tabular}{llll}
\hline Docente & 43 & 13 & 56 \\
Administrativo & 36 & 30 & 66 \\
Estudiantes & 361 & 334 & 695 \\
Total & 440 & 337 & 817 \\
\hline Fuente: & \\
\hline
\end{tabular}

\section{Validación}

En la investigación el cuestionario que se aplicó sirvió para medir lo que realmente se quería medir, existe validez de contenido, de criterio y de constructo. La variable a medir está relacionada a las dimensiones, indicadores e ítems respectivos.

Tabla 2. Calificación del instrumento de la validez de contenido a través de juicio de expertos de la variable: Calidad educativa

\begin{tabular}{ccccc}
\hline $\mathrm{N}^{\mathrm{o}}$ & Experto & Especialidad & Calificación Instrumento & Cant. \\
\hline Experto 1 & Dra. Ñanez Silva Miriam Viviana & Metodóloga & Suficiencia & 1 \\
Experto 2 & Dr. José Gabriel Chahuara Ardiles & Metodólogo & Suficiencia & 1 \\
Experto 3 & Dr. Sánchez Ortega Jaime Agustín & Temático & Suficiencia & 1 \\
& & & Suficiencia & 3 \\
\hline
\end{tabular}

\section{Confiabilidad}

Para verificar la confiabilidad del instrumento se aplicó el coeficiente de confiabilidad de Alfa de Cronbach, y se registra un alto índice de fiabilidad:

Tabla 3. Confiabilidad del instrumento: Calidad educativa

\begin{tabular}{ccc}
\hline Alfa de Cronbach & $\mathrm{N}^{\circ}$ de elementos \\
\hline &, 904 & 35 \\
\hline
\end{tabular}

Se ha elaborado un cuestionario polinómico con respuestas de tipo escala de Likert con un total de 35 ítems, distribuido en cuatro dimensiones: gestión estratégica, formación integral, soporte institucional y resultados.

\section{Métodos de análisis de datos}

Para realizar el análisis de los datos obtenidos en la encuesta se realizará la tabulación estadística y posteriormente se procederá al análisis comparativo explicativo, en cuadros, 
gráficos, así como tablas unidimensionales y bidimensionales. En la investigación debido al número de la muestra se aplicará la prueba de U Mann - Whitney, esta es una prueba no paramétrica, aplicada a dos muestras independientes. Esta prueba es tal, que da mayor peso porque muestra diferencia grande entre las dos condiciones que a la par se exhibe en una diferencia pequeña (Namakforoosh, 2014, p.348). En el análisis estadístico se utilizaron indicadores como la media, mediana, moda, desviación estándar, varianza, etc.; con un nivel de confianza alto, haciendo uso de los programas Excel y SPSS versión 22.

\section{Resultados}

La hipótesis en la investigación busco determinar si la aplicación del programa de innovación "Rumbo al licenciamiento", tiene efectos significativos en la gestión educativa, para la mejora de la calidad educativa en la carrera de administración de la universidad nacional de Cañete. Dimensionado al contexto de la reforma educativa y la política de aseguramiento de la calidad de la educación superior Universitaria en el país, se consideró que los directivos de la universidad de Cañete, están tomando conciencia de la importancia de la gestión estratégica y reorientando la gestión educativa hacia el cumplimiento de las dimensiones, reafirmando la importancia en la gestión de las personas para alcanzar los objetivos institucionales de la universidad y garantizar el aseguramiento de la calidad educativa.

A continuación, se presenta los resultados después de la aplicación del programa de innovación "rumbo al licenciamiento", en la gestión educativa en la mejora de la calidad educativa en la carrera de administración de la universidad nacional de Cañete en el año 2015. Para verificar si el programa tuvo éxito se realizó el análisis estadístico en dos momentos; en primera instancia a la presentación descriptiva, donde las puntuaciones de cada dimensión fueron trasformadas en niveles y luego en el análisis de la prueba de hipótesis correspondiente.

Tabla 4. Distribución de frecuencia de los resultados de la aplicación del programa de innovación en la calidad educativa

\begin{tabular}{|c|c|c|c|c|c|c|c|}
\hline & & & \multicolumn{4}{|l|}{ TEST } & \multirow[t]{2}{*}{ Total } \\
\hline & & & $\begin{array}{l}\text { Pre } \\
\text { control }\end{array}$ & $\begin{array}{l}\text { Pre } \\
\text { experimental }\end{array}$ & $\begin{array}{l}\text { Post } \\
\text { control }\end{array}$ & $\begin{array}{l}\text { Post } \\
\text { experimental }\end{array}$ & \\
\hline \multirow{6}{*}{$\begin{array}{l}\text { Calidad } \\
\text { educativa }\end{array}$} & Aceptable & Recuento & 47 & 2 & 50 & 0 & 99 \\
\hline & & $\begin{array}{ll}\% & \text { del } \\
\text { total } & \end{array}$ & $16,5 \%$ & $0,7 \%$ & $17,6 \%$ & $0,0 \%$ & $34,9 \%$ \\
\hline & Buena & Recuento & 24 & 53 & 21 & 39 & 137 \\
\hline & & $\begin{array}{ll}\% & \text { del } \\
\text { total } & \end{array}$ & $8,5 \%$ & $18,7 \%$ & $7,4 \%$ & $13,7 \%$ & $48,2 \%$ \\
\hline & Muy & Recuento & 0 & 16 & 0 & 32 & 48 \\
\hline & buena & $\begin{array}{ll}\% & \text { del } \\
\text { total } & \end{array}$ & $0,0 \%$ & $5,6 \%$ & $0,0 \%$ & $11,3 \%$ & $16,9 \%$ \\
\hline \multirow[t]{2}{*}{ Total } & & Recuento & 71 & 71 & 71 & 71 & 284 \\
\hline & & $\begin{array}{ll}\% & \text { del } \\
\text { total } & \\
\end{array}$ & $25,0 \%$ & $25,0 \%$ & $25,0 \%$ & $25,0 \%$ & $100,0 \%$ \\
\hline
\end{tabular}




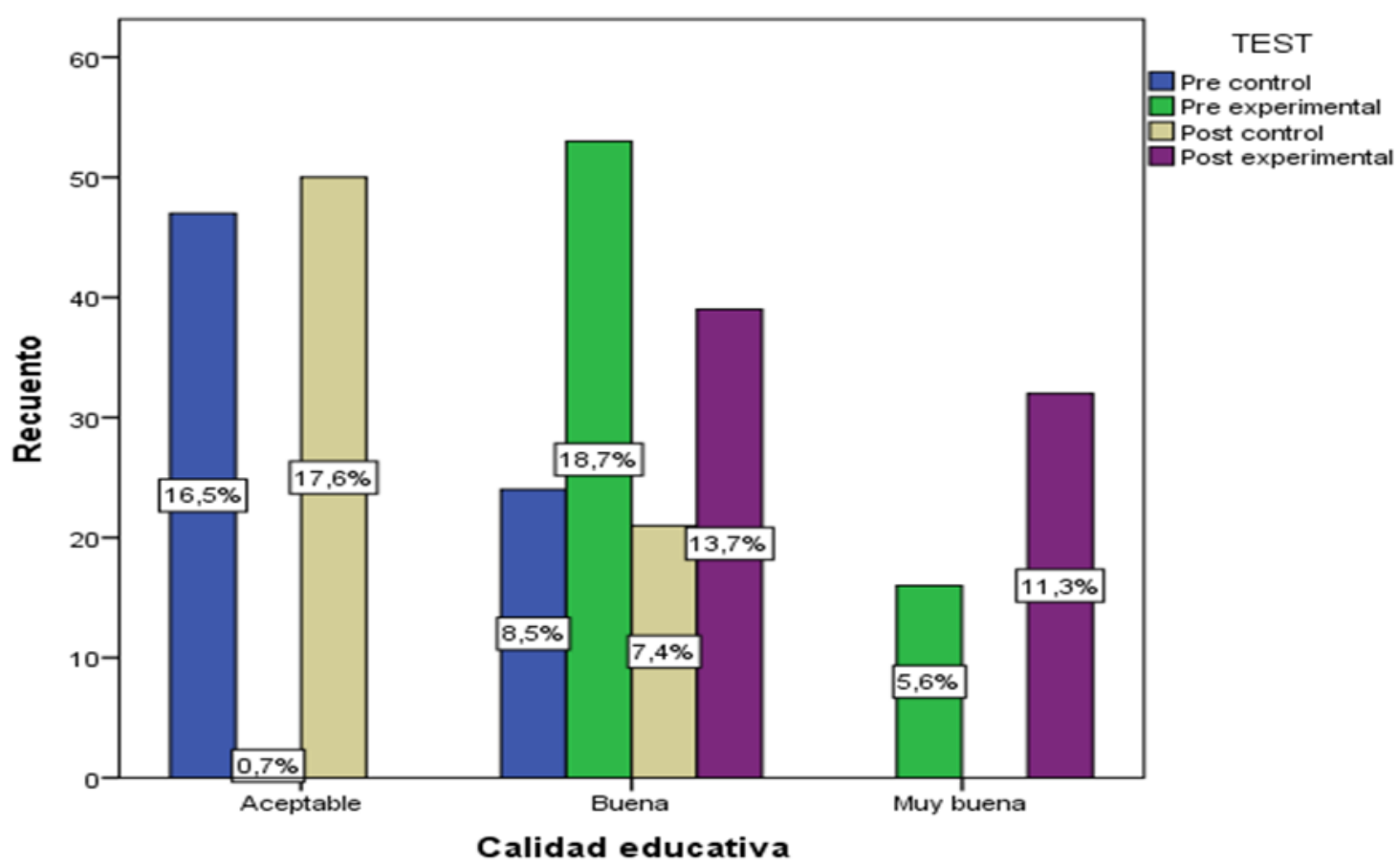

Figura 2. Comparación de los niveles de la calidad educativa por test

En la tabla, se aprecian los calificativos por test expresados en niveles, donde se observan las comparaciones entre el pre y post test, donde el 16,5\% de los informantes del grupo control en el pre test han manifestado que existe una calidad educativa aceptable, mientras que el 18,7\% del grupo experimental han referido que la calidad educativa está en un nivel buena, luego de la aplicación del programa de innovación "rumbo al licenciamiento", para mejorar el nivel de la calidad educativa, se obtuvo como resultado del post test en el grupo control que el 17,6\% de los informantes han referido que la calidad está en un nivel aceptable mientras que el 13,7\% anuncia que la calidad es buena y otros 11,3\% del grupo experimental manifiestan que el nivel de la calidad educativa está en un nivel muy buena en la carrera de administración de la universidad nacional de Cañete en el año 2015.

\section{Análisis descriptivo}

En cuanto a la figura se tiene el diagrama de frecuencias, donde se aprecia el comportamiento de los niveles por grupos de investigación, se aprecia que en el pre control y en el pre experimental, un grupo mayoritario se ubica en nivel aceptable, sin embargo después de la aplicación del programa se observa en el grupo experimental se observa la existencia de un nivel muy bueno de la calidad de gestión estratégica en la carrera de administración de la universidad nacional de Cañete en el año 2015. La aplicación del programa de innovación "Rumbo al licenciamiento", tiene efectos significativos en la calidad educativa de los estudiantes de la carrera de administración de la universidad Nacional de Cañete en el año 2015. 


\section{Análisis Inferencial}

- Ho: La aplicación del programa de innovación educativa "rumbo al licenciamiento", no tiene efectos significativos en la calidad educativa de los estudiantes de la carrera de administración de la Universidad Nacional de Cañete.

$$
\text { Ho: } \mu 1=\mu 2
$$

- H1: La aplicación del programa de innovación educativa "rumbo al licenciamiento", tiene efectos significativos en la calidad educativa de los estudiantes de la carrera de administración de la Universidad Nacional de Cañete.

$$
\text { Hi: } \mu 1>\mu 2
$$

\section{Conclusiones}

Primera:

Los resultados y valores inferenciales de la comparación entre el pre test del control y experimental nos indica que existe diferencia luego del experimento de la comparación entre el post test donde se tiene, el valor de la z se encuentra por debajo del nivel crítico zc $<-1,96$ y el $p=0,000$ menor al $\alpha 0,05$ por lo que se concluye que la aplicación del programa de innovación "Rumbo al licenciamiento", tiene efectos significativos en la calidad educativa de los estudiantes de la carrera de administración de la universidad nacional de Cañete en el año 2015.

Segunda:

De los resultados y valores inferenciales de la comparación entre el pre test del control y experimental, existe diferencia después del experimento entre el post test donde tiene el valor de $\mathrm{z}$ que se encuentra por debajo del nivel crítico zc $<-1,96$ y el $\mathrm{p}=0,000$ menor al $\alpha 0,05$ lo que se llega a la conclusión que la aplicación del programa de innovación "Rumbo al licenciamiento", tiene efectos significativos en la calidad educativa en la dimensión gestión estratégica en la carrera de administración de la Universidad Nacional de Cañete en el año 2015.

Tercera:

De los resultados y valores inferenciales de la comparación entre el pre test del control y experimental, existe diferencia después del experimento entre el post test donde tiene, el valor de $\mathrm{z}$ que se encuentra por debajo del nivel crítico $\mathrm{zc}<-1,96$ y el $\mathrm{p}=0,000$ menor al $\alpha 0,05$ lo que se llega a la conclusión que la aplicación del programa de innovación "Rumbo al licenciamiento", tiene efectos significativos en la calidad educativa en la dimensión formación integral de los estudiantes de la carrera de administración de la Universidad Nacional de Cañete en el año 2015. 
Cuarta:

De los resultados y valores inferenciales que se muestran en la tabla, la comparación entre el pre test del control y experimental no existe diferencia alguna luego del experimento la comparación entre el post test se tiene, el valor de la z se encuentra por debajo del nivel crítico zc $<-1,96$ y el $p=0,000$ menor al $\alpha 0,05$ lo que significa rechazar la hipótesis nula y aceptar la hipótesis alterna, por lo que la aplicación del programa de innovación "Rumbo al licenciamiento", tiene efectos significativos en la calidad educativa: dimensión soporte institucional de los estudiantes de la carrera de administración de la Universidad Nacional de Cañete en el año 2015.

Quinta:

De los resultados y valores inferenciales que se muestran en la tabla, la comparación entre el pre test del control y experimental no existe diferencia alguna luego del experimento la comparación entre el post test se tiene, el valor de la z se encuentra por debajo del nivel crítico zc $<-1,96$ y el $p=0,000$ menor al $\alpha 0,05$ lo que significa rechazar la hipótesis nula y aceptar la hipótesis alterna, por lo que la aplicación del programa de innovación "Rumbo al licenciamiento", tiene efectos significativos en la calidad educativa: resultados de los estudiantes de la carrera de administración de la Universidad Nacional de Cañete en el año 2015.

\section{Agradecimientos}

Al Dr. Jaime Agustín Sánchez Ortega, por su apoyo profesional y transmisión de oportunidades de crecimiento profesional, en mi carrera.

Al Dr. José Gabriel Chahuara Ardiles, Vicepresidente académico de la UNDC por su apoyo constante en la aplicación y consultas para concluir con la investigación.

A las autoridades, docentes, administrativos y estudiantes de la Universidad Nacional de Cañete, por su apoyo en este trabajo de investigación.

\section{Bibliografía}

Cataño, R. (2005). La Gestión integral de recursos humanos. Alcalá de Henares, España: Universidad de Alcalá.

Centro Interuniversitario de Desarrollo (CINDA). (2012). Aseguramiento de la calidad en Iberoamérica., (pág. 97).

Cesar Barzola, L. (2012). Gestión del recurso humano en enfermería. Tesis, Universidad de Nacional de Cuyo, Escuelas de enfermería, Facultad de Ciencias Médicas, Mendoza. Recuperado el 10 de marzo de 2016.

Martínez, A. C. (2015). Los desafíos para la Dirección de Recursos Humanos en el próximo quinquenio. En Capital Humano (págs. 65-75). España. Obtenido de www.capitalhumano.es 
Méndez Valenzuela, C. A. (mayo-junio de 2009). Reflexión sobre la planificación de los recursos humanos y la autonomía de la gestión en los hospitales de Chile. Revista Especializada en Salud Pública, 371-378. Recuperado el 27 de marzo de 2016.

Ministerio de Educación. (2015). Política de aseguramiento de la calidad de la educación Superior Universitaria. Lima.

Ministerio de Educación, DIGESU. (2015). Condiciones Básicas de Calidad. Lima: MINEDU.

Morales Fernández, E., Ariza Montes, A., \& Morales Gutiérrez, A. (12 de diciembre de 2013). La evolución de la gestión de recursos humanos desde una perspectiva estratégica. Revista de Fomento Social - RFS, 309-329. doi:ISSN 00156043.

Pérez, R. (2015). Hacia una educación de calidad, gestión e instrumentos y evaluación. Avances de supervisión educativa. Recuperado el 2015 de noviembre de 30, de http://dialnet.unirioja.es/servlet/revista? codigo $=8645$

SUNEDU. (28 de marzo de 2016). http://www.sunedu.gob.pe/. Obtenido de http://www.sunedu.gob.pe/: http://www.sunedu.gob.pe/dilic/ 